\title{
Use of Accelerometry to Monitor Physical Activity in Critically Ill Subjects: A Systematic Review
}

\author{
Avelino C Verceles MD and Erin R Hager PhD
}

\author{
Introduction \\ Methods \\ Eligibility Criteria \\ Results \\ Discussion \\ Future Directions
}

\begin{abstract}
Medical management of critically ill patients often incorporates prolonged bed rest, which, in combination with the underlying illness, results in global muscle weakness and atrophy. Recent evidence has demonstrated improvements in clinical and functional outcomes when exercise and physical activity are incorporated early in the management of ICU patients. Accurate monitoring of ICU patients' physical activity is essential for proper prescription and escalation of activity levels. Accelerometry is a technique used to measure physical activity and has been validated in several ambulatory populations. However, its use in critically ill, hospitalized patients with poor functional mobility is limited. In this review, we focus on the few studies assessing the use of accelerometry to measure physical activity in the care of mechanically ventilated adult ICU patients. The selected literature demonstrates that accelerometry correlates well with direct observation in reporting frequency and duration of various types of physical activity (rolling, sitting up, transferring, walking), but cannot differentiate various intensities of activity or whether movements are voluntary or involuntary with respect to effort. Thus, although accelerometry may serve as a useful adjunct in reporting temporality of physical activity in critically ill patients, other objective information may be needed to accurately record frequency, duration, and intensity of activity in this population. Key words: critical care; accelerometry; actigraphy. [Respir Care 2015;60(9):1330-1336. ( 2015 Daedalus Enterprises]
\end{abstract}

\section{Introduction}

Over 5 million patients are admitted to ICUs annually in the United States, ${ }^{1}$ with the most common diagnoses being

\footnotetext{
Dr Verceles is affiliated with the Division of Pulmonary, Critical Care, and Sleep Medicine, and Dr Hager is affiliated with the Department of Pediatrics, University of Maryland School of Medicine, Baltimore, Maryland.

Dr Verceles was supported by National Institute on Aging Grant R03 AG045100 and a Grant for Early Medical/Surgical Subspecialists in Transition to Aging Research (GEMSSTAR). The authors have disclosed no conflicts of interest.
}

respiratory failure, postoperative management, ischemic heart disease, sepsis, and heart failure. ${ }^{2}$ Given their severity of illness, these patients are often treated with full-time bed rest due to hemodynamic instability, need for mechanical ventilation, or sedation requirements to treat agitation. Thus, patients in ICUs can experience weeks of bed rest

\footnotetext{
Correspondence: Avelino C Verceles MD, Division of Pulmonary, Critical Care, and Sleep Medicine, 110 South Paca Street, Second Floor, Baltimore, MD 21201. E-mail: avercele@medicine.umaryland.edu.
}

DOI: $10.4187 /$ respcare. 03677 
during the course of their stay, which results in a high proportion of these patients developing ICU-acquired weakness and muscle atrophy. ${ }^{3-5}$

The incorporation of exercise and physical activity early in the course of critical illness results in improved clinical outcomes, including lower incidence of ICU-acquired weakness, faster weaning from mechanical ventilation, lower incidence of delirium, and decreased ICU stay. ${ }^{6,7}$ Despite various barriers to the incorporation of exercise strategies in the care of critically ill patients (physical barriers such as central lines, feeding tubes, and urinary catheters and cultural barriers such as staff reluctance to exercise critically ill patients), exercise and physical activity are gradually becoming accepted as the standard of care for ICU patients. Specific exercise interventions vary in intensity and duration and range from brief, basic mobility movements (rolling, sitting at the edge of a bed, transferring) to pre-ambulation maneuvers (sitting-tostanding, pivoting) and extended ambulatory (timed walking) trials. Given the effects of therapeutic exercise and physical activity in this population, knowing the exact duration and intensity of a patient's physical activity may be helpful to clinicians when prescribing exercise regimens to be incorporated into the plan of care.

Accurately measuring physical activity is important, as it allows clinicians to determine patient-specific peak efforts with respect to frequency, duration, and intensity. Additionally, measuring physical activity allows one to examine trends and correlates of activity, all of which may allow for proper prescription of an exercise regimen and for appropriate escalation of physical activity goals. Studies quantifying physical activity in the ICU most commonly utilize direct observation, as other means (self-report, ecological momentary assessment) may not be feasible due to patient incapacitation from critical illness or sedation. The use of direct observation in measuring physical activity in the critically ill population requires a trained observer to document the activity of each patient. Thus, observation is expensive, as it involves training and compensating the observer. Additionally, the amount of observations may be restricted by the number of observers employed, introducing a limiting factor to measuring physical activity.

Accelerometry is a technique used to measure activity and has been validated in several ambulatory populations. ${ }^{8-10}$ It entails the use of an accelerometer, a small device that can sense, count, and record movement. The accelerometer is usually worn on a patient's wrist or ankle, and it collects movement data continuously. Accelerometry is very accurate and allows for uninterrupted measurements of physical activity as long as the device is worn. ${ }^{10}$ Because the accelerometer is unobtrusive and does not limit normal physical performance, patients can wear an accelerometer and not feel as if they are being ob- served, allowing them to conduct their activities as usual. Although accelerometry appears to be a simple, accurate, and concise method to measure physical activity, its use in studies to monitor critically ill patients has been limited. Because there are so few studies utilizing this technology, the true accuracy of accelerometry in measuring physical activity in ICU patients in unknown.

This aim of this study was to describe how accelerometry has been used to measure physical activity in critically ill, mechanically ventilated subjects. Accelerometry was chosen because it is a simple, inexpensive assessment procedure that provides a considerable amount of information and has been well validated in several populations as an effective means of quantifying and characterizing physical activity. ${ }^{11-13}$ Specifically, we compare accelerometry with other methods of physical activity assessment that have been used in the critically ill population (activity logs, observation, nursing documentation). The assessment of activity and mobility in critically ill patients is a topic of increasing interest, as early mobility and physical rehabilitation in this population have been shown to dramatically improve clinical outcomes.

\section{Methods}

We performed a PubMed search for relevant trials using the following search terms: critical care and accelerometry, critical care and actigraphy, mechanical ventilation and accelerometry, and mechanical ventilation and actigraphy. All abstracts were reviewed, and trials were selected if they met eligibility criteria.

\section{Eligibility Criteria}

Eligibility criteria for each trial were: admittance to an ICU (medical, surgical, cardiac, cardiothoracic) or mechanical ventilatory support (designating subjects as critically ill), adult ( $>18 \mathrm{y}$ of age), all 4 limbs intact, and use of an accelerometer worn on the wrist or ankle to measure or monitor physical activity or sleep/wake-related physical activity. Trials were excluded if they were written in languages other than English, did not involve subjects admitted to ICUs or receiving mechanical ventilation, did not include accelerometry or actigraphy in the methods, included subjects with catastrophic neurologic events (intracranial hemorrhage, stroke, traumatic brain injury), or did not focus on human subjects. Given the novelty of accelerometry in monitoring physical activity in hospitalized patients, we chose to review all studies regardless of study date.

\section{Results}

The literature search yielded a total of 104 manuscripts: critical care and accelerometry, 29; critical care and actig- 


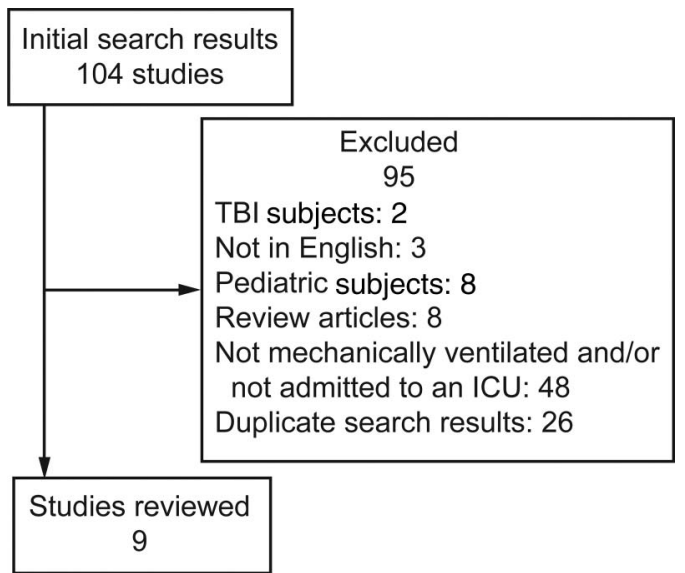

Fig. 1. Literature search flow chart. $\mathrm{TBI}=$ traumatic brain injury.

raphy, 34; mechanical ventilation and accelerometry, 17; and mechanical ventilation and actigraphy, 24. After elimination of duplicate studies in all searches and review of all abstracts, 9 primary resources met the inclusion criteria and were included in the review (Fig. 1). Summaries of all primary resources are provided in Table 1 .

We reviewed 9 studies written by 5 different author groups. Two author groups each wrote 3 articles that met the inclusion criteria. Eight studies focused on adult medical and surgical ICUs, and one study focused on mechanically ventilated subjects in a post-acute care weaning unit. In all 9 studies, at least a proportion of subjects received mechanical ventilation, with $100 \%$ of patients receiving ventilatory support in 7 studies.

All of the studies were prospective, with 4 studies using the Motionlogger (Ambulatory Monitoring, Ardsley, New York) to monitor and measure activity. In 4 of the 9 studies, 1-min epochs were used as a monitoring resolution, whereas 12-30-s epochs were used in 5 studies. All studies correlated the measured activity with another outcome or correlated the accelerometry-measured physical activity with other assessment techniques (Table 2). In 6 of the 9 studies, subjects wore the accelerometer on their wrists, whereas in 3 studies, subjects wore accelerometers on their wrists and ankles.

Four studies used accelerometry as a measurement strictly of activity. ${ }^{14-17}$ Three studies used accelerometry to measure physical activity and movement as surrogates for adequacy of sedation, ${ }^{18-20}$ and 2 studies used accelerometry as a surrogate for measuring sleep/wake cycles. ${ }^{21,22}$

In the 4 studies that used accelerometry as a measurement strictly of activity, ${ }^{14-17}$ all noted that accelerometry accurately measured duration of physical activity, but were not able to distinguish intensity. More specifically, accelerometry was not able to distinguish between voluntary or volitional movements versus passive range of motion, information that is of great importance when performing studies focused on physical activity. One study that assessed the agreement between accelerometry and observation noted that the Motionlogger accelerometer overestimated physical activity by 25\% (indicating 261 activity occurrences when only 196 occurred). ${ }^{14}$ This study was limited, however, by its design, specifically the incorporation of only two 4-h observation periods during which an observer actively logged activity. Thus, continuous observation of these subjects in the native ICU environment was not performed.

Two of these 4 studies associated physical activity with inflammatory cytokine levels and activity levels over a short time period (24-48 h) and compared the activity with direct observation. ${ }^{14,15}$ Both studies demonstrated the association of low-level physical activity with decreasing cytokine levels. Agreement between direct observation and accelerometry was $90 \%$ for duration and $89 \%$ for frequency in these 2 studies. Although the short period of physical activity monitoring may have been adequate for the study, $48 \mathrm{~h}$ of accelerometry assessment would not be considered adequate to draw a real-life conclusion regarding daily physical activity in this cohort of ICU subjects.

In the fourth study by Mistraletti et al, ${ }^{17}$ each subject was required to wear the accelerometer for the entire ICU stay, and activity levels were measured and correlated to depth of sedation per objective nursing assessment. This study found a strong relationship between physical activity, as measured by accelerometry, and depth of sedation, as rated by the Richmond Agitation-Sedation Scale, and concluded from these findings that accelerometry should be considered for monitoring sedation in the ICU. Although all 4 of the studies had small sample sizes, ${ }^{14-17}$ this study had the fewest number of subjects $(N=13) .{ }^{17}$ Thus, the statement advocating accelerometry as a standard for monitoring sedation may be too broad a generalization without a large definitive trial.

Three studies correlated physical activity and movement with adequacy of sedation, indicating less movement as more sedated or more comfortable compared with more movement or less adequately sedated. ${ }^{18-20}$ These studies enrolled adult medical, cardiac, and surgical ICU subjects and used different accelerometers (Actiwatch 16 [Philips Respironics, Murrysville, Pennsylvania] in one study and Motionlogger in the other two studies). In an effort to improve physical activity measurement accuracy, the subjects in these studies wore 2 accelerometers, one each on their wrists and ankles. Shorter epoch lengths were used, which also increased accuracy in physical activity measurement. The major findings in these 3 studies were: (1) accelerometry correlated with sedation, as measured by the Richmond Agitation-Sedation Scale; (2) comfort (movement suppression) was not uniformly achieved with stable physiology and sedation; and (3) movement suppression was achievable through sedation, whereas physiologic stress 


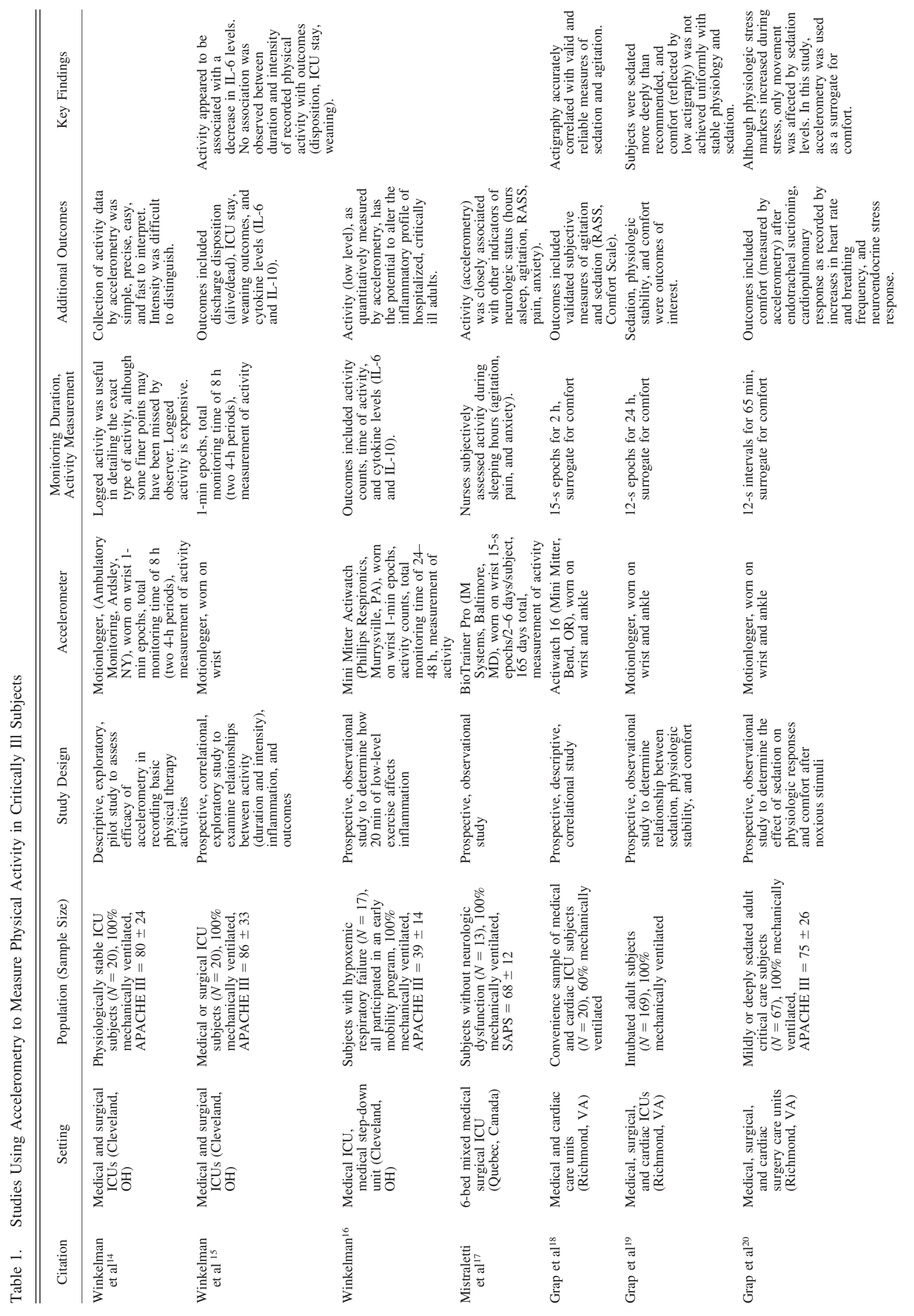




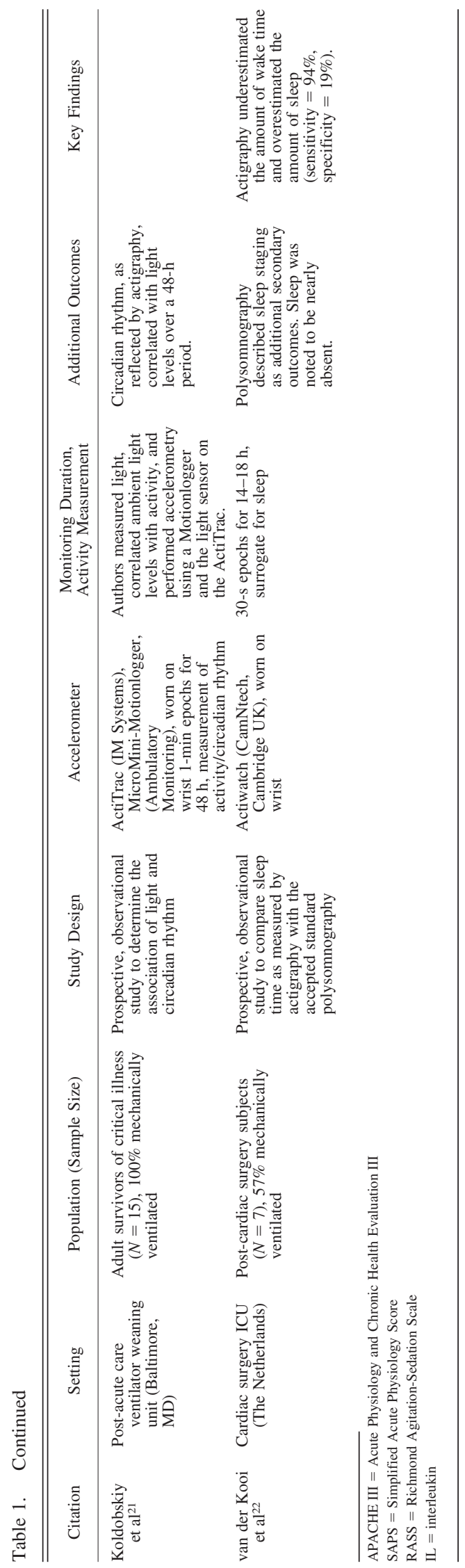

Table 2. Outcomes and Other Variables Associated With Accelerometry

\begin{tabular}{lc}
\hline \hline \multicolumn{1}{c}{ Outcome/Covariate } & $\begin{array}{c}\text { No. of Studies Comparing } \\
\text { Covariate and Accelerometry }\end{array}$ \\
\hline Sedation/subjective assessment/logs & 4 \\
Comfort & 2 \\
Inflammation/cytokines & 2 \\
Sleep/wake/circadian rhythm & 2 \\
Neuroendocrine stress response & 1 \\
Cardiopulmonary response & 1 \\
\hline
\end{tabular}

suppression in response to stimuli was not. These 3 studies ${ }^{18-20}$ had larger samples sizes $(N=20,169$, and 67, respectively) compared with the first 4 studies $^{14-17}(N=20$, 20,17 , and 13 , respectively), but were all performed by the same investigator. Thus, an inherent bias may be present in these works.

The last 2 studies by Koldobskiy et $\mathrm{a}^{21}$ and van der Kooi et al ${ }^{22}$ used accelerometry as a surrogate for measuring sleep/wake cycles. Both studies evaluated accelerometry in clinically distinct populations: post-acute, ventilator-dependent subjects ${ }^{21}$ and acutely ill, postoperative cardiac surgery subjects. ${ }^{22}$ Both had small sample sizes ( $N=15$ and 7, respectively) and quantified sleep by decreased accelerometric activity. Koldobskiy et al ${ }^{21}$ correlated activity level with ambient light. Two accelerometers were used in this study: one worn on the subject's wrist to detect physical activity and the other with light-sensing capability posted at the head of the subject's bed at eye level. It was determined that physical activity measured by accelerometry followed a diurnal circadian pattern that was closely associated with the diurnal pattern of ambient sunlight inherent to the ventilator unit. Although this study incorporated a short epoch length and a modest monitoring period duration, the subjects were all survivors of prolonged ICU stays. Thus, one cannot rule out the possibility of ICU-acquired weakness, which would have affected the accuracy of accelerometer measurements by underestimating wake time due to decreased volitional movement.

van der Kooi et $\mathrm{al}^{22}$ compared the use of accelerometry for sleep measurement and overnight polysomnography, the accepted standard of sleep assessment. A standard duration of $30 \mathrm{~s}$ was used to track movement during accelerometry, with monitoring periods of $14-18 \mathrm{~h}$ in duration. Accelerometry was found to underestimate the amount of wake time and overestimate the amount of sleep compared with polysomnography, with a dismally low specificity of $19 \%$. The authors concluded that accelerometry should not be used for sleep measurement and assessment in critically ill patients, despite the small sample size $(N=7)$ and the specialized cardiac surgery subject population, 2 concerns of the study that may have affected its generalizability. 


\section{Use of AcCelerometry in CRItically Ill SubJects}

\section{Discussion}

In this review, we found that accelerometry has often been used to measure movement as a surrogate for other outcomes (eg, sedation, comfort, sleep). This may be attributed, in part, to the fact that, until recently, few ICU staff felt comfortable exercising their patients or allowing them to participate in any physical activity. In 5 of these 9 studies, physical activity measured by accelerometry was compared with activity subjectively reported by an observer (movement, agitation, comfort, sleep). Strong positive correlations were found between physical activity and agitation and stress response in unsedated patients, whereas significant negative associations were found between physical activity and comfort level, depth of sedation, and hours of sleep. The recent evolution of ICU culture $^{23,24}$ toward incorporating exercise and physical activity early in the management plan of critically ill patients may result in more studies using accelerometry strictly for physical activity measurement, rather than as a surrogate for other outcomes. Thus, the use of accelerometry to monitor baseline activity provides a uniform method for physical therapists, nursing staff, and other care providers to track exercise and rehabilitation progress.

Accelerometry is a simple means of objectively measuring physical activity and has been well validated in numerous populations. As the management paradigm of critically ill patients shifts toward incorporating early mobility and increased physical activity, it seems logical to include a straightforward quantitative assessment of physical activity in the monitoring of such patients. Accelerometry fills this need, as it is inexpensive, requires little effort, collects continuous current data, and is not obtrusive. Additionally, accelerometry can be used to quantify sleep, ${ }^{21,22}$ comfort, ${ }^{18-20}$ and adequacy of sedation ${ }^{17}$ when monitoring critically ill patients. Given the multitude of monitoring devices already present in ICUs, the incorporation of accelerometry would require minimal effort and cost, yet yield reliable and valid information regarding activity.

Citing the limitations of this review, first, most of the studies included very small sample sizes, with 7 of the 9 studies enrolling $\leq 20$ subjects. Thus, the conclusions drawn from the accelerometry tracings may not accurately represent the outcome of interest across broader populations. Second, the monitoring periods were short, ranging from $65 \mathrm{~min}$ to $48 \mathrm{~h}$. This may have led to a false representation of the total physical activity performed during the course of an ICU stay, especially if the subject was uncharacteristically overactive or sedentary during the monitoring period. Third, only 4 of the studies used accelerometry to measure physical activity, whereas 5 studies used accelerometry as a surrogate to quantify comfort and sedation levels or to monitor sleep or circadian rhythm. Finally, our literature search was limited to a single database (PubMed), which may appear to limit the number of articles that met our inclusion criteria. In actuality, PubMed is the only medical database that incorporates optimal update frequency and includes online early articles, which allowed us to include the most recent, up-to-date literature in our review. ${ }^{25}$

\section{Future Directions}

As exercise and physical activity evolve into cornerstones in the management of critically ill patients, accurate monitoring of duration, frequency, and intensity of all therapeutic movements will increase in importance. Although accelerometry can satisfactorily monitor duration and frequency of physical activity, its accuracy in measuring intensity is limited. Although other uses for accelerometry as a monitoring modality have shown some promise (monitoring levels of comfort and agitation, depth of sedation), the results obtained by monitoring these outcomes are best interpreted in combination with a subjective assessment of a caregiver. Although not considered the standard of care at this time, the use of accelerometry in the routine care of ICU patients would allow more accurate characterization of physical activity (ie, voluntary, independent movement vs non-volitional, assisted movement such as bathing or rolling performed by a nurse) compared with present methods. Thus, as the care paradigm of ICU patients shifts toward the incorporation of early mobility and exercise protocols, we believe that further studies investigating the use of accelerometry in monitoring the activity of critically ill patients should focus on combining it with periodic bedside observation to allow more accurate assessments of activity intensity, frequency, and duration.

\section{REFERENCES}

1. Committee on the Future Health Care Workforce for Older Americans, Institute of Medicine of the National Academies. (2015) Retooling for an aging America: building the health care workforce. Washington, DC: The National Academies Press.

2. Society of Critical Care Medicine. Critical care statistics. www.sccm.org/Communications/Pages/CriticalCareStats.aspx. Accessed April 29, 2014.

3. De Jonghe B, Sharshar T, Lefaucheur JP, Authier FJ, Durand-Zaleski I, Boussarsar M, et al. Paresis acquired in the intensive care unit: a prospective multicenter study. JAMA 2002;288(22):28592867.

4. Coakley JH, Nagendran K, Yarwood GD, Honavar M, Hinds CJ. Patterns of neurophysiological abnormality in prolonged critical illness. Intensive Care Med 1998;24(8):801-807.

5. de Letter MA, Schmitz PI, Visser LH, Verheul FA, Schellens RL, Op de Coul DA, van der Meché FG. Risk factors for the development of polyneuropathy and myopathy in critically ill patients. Crit Care Med 2001;29(12):2281-2286.

6. Schweickert WD, Pohlman MC, Pohlman AS, Nigos C, Pawlik AJ, Esbrook CL, et al. Early physical and occupational therapy in mechanically ventilated, critically ill patients: a randomised controlled trial. Lancet 2009;373(9678):1874-1882. 


\section{Use of AcCelerometry in CRITICAlly Ill SubJects}

7. Morris PE, Goad A, Thompson C, Taylor K, Harry B, Passmore L, et al. Early intensive care unit mobility therapy in the treatment of acute respiratory failure. Crit Care Med 2008;36(8):2238-2243.

8. Meijer GA, Westerterp KR, Verhoeven FM, Koper HB, ten Hoor F. Methods to assess physical activity with special reference to motion sensors and accelerometers. IEEE Trans Biomed Eng 1991;38(3): 221-229.

9. Nichols JF, Morgan CG, Sarkin JA, Sallis JF, Calfas KJ. Validity, reliability, and calibration of the Tritrac accelerometer as a measure of physical activity. Med Sci Sports Exerc 1999;31(6):908-912.

10. Matthews CE, Freedson PS. Field trial of a three-dimensional activity monitor: comparison with self report. Med Sci Sports Exerc 1995;27(7):1071-1078.

11. Ekblom O, Nyberg G, Bak EE, Ekelund U, Marcus C. Validity and comparability of a wrist-worn accelerometer in children. J Phys Act Health 2012;9(3):389-393.

12. Brown DK, Grimwade D, Martinez-Bussion D, Taylor MJ, Gladwell VF. The validity of the ActiPed for physical activity monitoring. Int J Sports Med 2013;34(5):431-437.

13. Taylor LM, Klenk J, Maney AJ, Kerse N, MacDonald BM, Maddison R. Validation of a body-worn accelerometer to measure activity patterns in octogenarians. Arch Phys Med Rehabil 2014;95(5):930934.

14. Winkelman C, Higgins PA, Chen YJ. Activity in the chronically critically ill. Dimens Crit Care Nurs 2005;24(6):281-290.

15. Winkelman C, Higgins PA, Chen YJ, Levine AD. Cytokines in chronically critically ill patients after activity and rest. Biol Res Nurs 2007;8(4):261-271.

16. Winkelman C. Investigating activity in hospitalized patients with chronic obstructive pulmonary disease: a pilot study. Heart Lung 2010;39(4):319-330.
17. Mistraletti G, Taverna M, Sabbatini G, Carloni E, Bolgiaghi L, Pirrone $\mathrm{M}$, et al. Actigraphic monitoring in critically ill patients: preliminary results toward an "observation-guided sedation." J Crit Care 2009;24(4):563-567.

18. Grap MJ, Borchers CT, Munro CL, Elswick RK Jr, Sessler CN. Actigraphy in the critically ill: correlation with activity, agitation, and sedation. Am J Crit Care 2005;14(1):52-60.

19. Grap MJ, Munro CL, Wetzel PA, Best AM, Ketchum JM, Hamilton VA, et al. Sedation in adults receiving mechanical ventilation: physiological and comfort outcomes. Am J Crit Care 2012;21(3):e53-e63.

20. Grap MJ, Munro CL, Wetzel PA, Ketchum JM, Hamilton VA, Sessler CN. Responses to noxious stimuli in sedated mechanically ventilated adults. Heart Lung 2014;43(1):6-12.

21. Koldobskiy D, Diaz-Abad M, Scharf SM, Brown J, Verceles AC. Long-term acute care patients weaning from prolonged mechanical ventilation maintain circadian rhythm. Respir Care 2014;59(4):518524

22. van der Kooi AW, Tulen JH, van Eijk MM, de Weerd AW, van Uitert MJ, van Munster BC, Slooter AJ. Sleep monitoring by actigraphy in short-stay ICU patients. Crit Care Nurs Q 2013;36(2):169173.

23. Needham DM, Korupolu R, Zanni JM, Pradhan P, Colantuoni E, Palmer JB, et al. Early physical medicine and rehabilitation for patients with acute respiratory failure: a quality improvement project. Arch Phys Med Rehabil 2010;91(4):536-542.

24. Needham DM, Korupolu R. Rehabilitation quality improvement in an intensive care unit setting: implementation of a quality improvement model. Top Stroke Rehabil 2010;17(4):271-281.

25. Falagas ME, Pitsouni EI, Malietzis GA, Pappas G. Comparison of PubMed, Scopus, Web of Science, and Google Scholar: strengths and weaknesses. FASEB J 2008;22(2):338-342. 